\title{
Effects of Trehalose and Sucrose on Human Sperm Motility, Vitality and Morphology after Cryopreservation
}

Manaphat Suksai, M.D., Kriengsak Dhanaworavibul, M.D., M.Med.Sci.

Department of Obstetrics and Gynecology, Faculty of Medicine, Prince of Songkla University, Songkhla 90110, Thailand.

Received 3 October 2018 • Revised 6 February 2019 • Accepted 14 February 2019 • Published online 18 March 2019

\section{Abstract:}

Objective: To compare human sperm motility, vitality and morphology after cryopreservation among sucrose, and different trehalose concentrations.

Material and Methods: A total number of 124 normozoospermic semen samples were collected. Each semen sample was divided into 4 portions, and cryopreserved in a human sperm-preserving medium along with cryoprotectants, including; 50 milimolar $(\mathrm{mM})$ trehalose, $100 \mathrm{mM}$ trehalose, $200 \mathrm{mM}$ trehalose and $50 \mathrm{mM}$ sucrose, respectively. All semen samples were frozen by using a vapor phase method. Post-thawed sperm motility, vitality and morphology were assessed. R program was used for data analysis. A p-value of $<0.05$ was considered statistically significant.

Results: Post-thawed semen evaluation indicated that $50 \mathrm{mM}$ trehalose was better than $50 \mathrm{mM}$ sucrose in all sperm parameters, which included progressive motility ( $p$-value $=0.037$, total motility $(p-$ value $<0.001)$, vitality $(p-v a l u e<0.001)$ and morphology ( $p$-value<0.001). The sperm parameters were not significantly different among $100 \mathrm{mM}$ trehalose, $200 \mathrm{mM}$ trehalose and $50 \mathrm{mM}$ sucrose.

Conclusion: The use of $50 \mathrm{mM}$ trehalose, as non-permeating cryoprotectant, showed superior post-thaw sperm parameters over sucrose, and other trehalose concentrations.

Keywords: cryopreservation, human sperm, sucrose, trehalose

Contact: Kriengsak Dhanaworavibul, M.D., M.Med.Sci.

Department of Obstetrics and Gynecology, Faculty of Medicine,

Prince of Songkla University, Hat Yai, Songkhla 90110, Thailand.

Email: dkrieng@yahoo.com
J Health Sci Med Res 2019;37(2):101-107 doi: 10.31584/jhsmr.201945 www.jhsmr.org 


\section{Introduction}

Sperm cryopreservation is an integral part in preserving male fertility. Although several cryopreservation methods have been developed, over several decades, they still involve significant loss of spermatozoa motility and viability. Human sperm cryopreservation is notoriously unsuccessful, and the $40.0-60.0 \%$ survival rates observed today are little in difference from those of 40 years ago. In contrast oocyte and embryo cryosurvival can be as high as $90.0 \%{ }^{1}$

There are two main factors of sperm death, after sperm has been frozen-thawed, including; intracellular ice crystal formation and osmotic shock. ${ }^{2}$ Intracellular water is the main component of any cell. Without cryoprotectants, when cells are frozen, intracellular water may become ice crystals, which can cause irreversible damage. Therefore, cryoprotectants should be used to prevent ice crystal formation. They can also help to maintain osmotic change, during the frozen-thawed process.

The selection of types of cryoprotectants, either permeating or non-permeating, is particularly important for the cryopreservation process. To date, sucrose, a nonpermeating cryoprotectant, is most commonly used in sperm cryopreservation ${ }^{3}$; however, studies demonstrated the benefits of using trehalose, as a non-permeating cryoprotectant, are limited.

The beneficial effects of using trehalose, on the post-thawed viability of mammalian sperm cells, has been reported in many studies. The studies in buffalo, boar, mouse, and goat showed that: trehalose improved sperm motility, compared with using cryoprotectant without trehalose. $^{4-8}$

Although there is a number of animal studies supporting that trehalose has a positive effect on sperm parameters, the evidence in human sperm is very limited. Therefore, to optimize cryopreservation protocol in human sperm, the objective of this study was to compare human sperm motility, vitality and morphology after cryopreservation, among sucrose and with different trehalose concentrations.

\section{Material and Methods}

\section{Study population}

Semen samples were obtained from healthy male donors (age range 20-45 years old), who came to the infertility clinic at Songklanagarind Hospital. One hundred and twenty-four samples with normal semen parameters were recruited into the study. The approval of the Prince of Songkla University Research Ethical Committee was obtained, prior to the study, and all subjects were informed with respect to this study.

\section{Semen samples}

All donators had maintained sexual abstinence from 3 to 5 days. After signing an informed consent, fresh, ejaculated semen samples were obtained by masturbation into sterile containers. After ejaculation, samples were allowed to liquefy for $30-60$ minutes, at $37^{\circ} \mathrm{C}$. Sperm count and motility were scored using Makler ${ }^{\circledR}$ counting chamber (Sefi medican instruments, Haifa, Israel) under a microscope according to World Health Organization criteria $2010 .^{9}$ Sperm vitality was determined using an eosin-nigrosin staining technique, while sperm morphology was assessed by using Kruger strict criteria. ${ }^{10}$ All semen analysis was performed by a well-trained obstetrics and gynecology resident.

\section{Reagents and media}

Human sperm preserving medium $\left(\right.$ HSPM) ${ }^{11}$ (1:1) was prepared in our laboratory as follows: 100 millimolar (mM) $\mathrm{NaCl}, 5.365 \mathrm{mM} \mathrm{KCL}, 2.72 \mathrm{mM} \mathrm{CaCl}, 0.492 \mathrm{mM}$ $\mathrm{MgCl}_{2}, 12.856 \mathrm{mM}$ sodium lactate, $0.321 \mathrm{mM} \mathrm{NaH2PO4,}$ 30.949 mM NaHCO3, 20 mM Hepes-free acid, 133.21 $\mathrm{mM}$ glycine, and $5.506 \mathrm{mM}$ glycerol plus $4.9 \mathrm{~g} /$ human 
albumin, $15.0 \%$ volume/volume glycerol, streptomycin $0.1 \mathrm{mM}$ and penicillin $\mathrm{G} 0.2 \mathrm{mM}$. The cryoprotectants including $50 \mathrm{mM}$ trehalose, $100 \mathrm{mM}$ trehalose and 200 $\mathrm{mM}$ trehalose were added in experimental groups, respectively; and $50 \mathrm{mM}$ sucrose was added in the control group.

\section{Freezing and thawing protocol}

Each semen sample was divided into 4 equal portions, and each portion was diluted 1:1, with a freezing medium consisting of varying concentrations of trehalose, to obtain a final concentration of trehalose of $50 \mathrm{mM}, 100$ $\mathrm{mM}$ and $200 \mathrm{mM}$ and $50 \mathrm{mM}$ of sucrose (control group). Sperm had been mixed and incubated, with the freezing medium for 10 minutes. After 10 minutes of incubation, each sample was loaded by sperm straw, and then held $15 \mathrm{~cm}$ above the surface of $\mathrm{LN}_{2}$, on a metal rack in the vapor phase for 15 minutes. The straws were finally plunged into $\mathrm{LN}_{2}$ at $-196{ }^{\circ} \mathrm{C}$ for storage. All semen samples were frozen for about a week. To thaw, each straw was quickly immersed into a $37{ }^{\circ} \mathrm{C}$ water bath. Straw content was expelled into a $5 \mathrm{ml}$ tube. Post-thawed sperm parameters were examined.

\section{Statistical analysis}

The variables were expressed as mean \pm S.D. The mean difference was calculated by subtracting means of post-thawed sperm motility, vitality and morphology by means of pre-freeze parameters for fresh specimens. Mean differences of all parameters were tested for data normality as well as the sperm parameters between three different trehalose concentrations, comparing to sucrose as control group were compared by the Wilcoxon signed-ranks test, in paired samples. $R$ program was used for data analysis. A p-value of less than 0.05 was considered statistically significant.

\section{Results}

The standard semen quality parameters, namely; progressive motility, total motility, vitality and morphology of spermatozoa in fresh semen, and after the frozenthawed process of the 124 samples, are shown in Table 1.

Table 1 Sperm parameters before and after, the frozen-thawed process

\begin{tabular}{|c|c|c|c|c|c|c|}
\hline \multirow{3}{*}{$\begin{array}{l}\text { Parameters } \\
(n=124)\end{array}$} & \multirow{3}{*}{$\begin{array}{l}\text { Pre-freeze } \\
\text { (mean } \pm \text { S.D.) }\end{array}$} & \multicolumn{4}{|c|}{ Post-thaw (mean \pm S.D.) } & \multirow{3}{*}{ P-value* } \\
\hline & & \multicolumn{2}{|l|}{ Sucrose } & \multicolumn{2}{|l|}{ Trehalose } & \\
\hline & & $50 \mathrm{mM}$ & $50 \mathrm{mM}$ & $100 \mathrm{mM}$ & $200 \mathrm{mM}$ & \\
\hline Volume (mL) & $4.0 \pm 1.1$ & & & & & \\
\hline Conc $\left(10^{6} / \mathrm{mL}\right)$ & $56.9 \pm 26.6$ & $24.3 \pm 16.1$ & $25.1 \pm 16.1$ & $24.5 \pm 16.1$ & $24.2 \pm 16.1$ & \\
\hline Total mot $(\%)$ & $73.2 \pm 9.6$ & $9.9 \pm 10.9 \S$ & $16.8 \pm 12.8$ & $11.3 \pm 12.8 \S$ & $7.6 \pm 9.5 \S$ & $<0.001$ \\
\hline PR (\%) & $50.6 \pm 11.8$ & $3.2 \pm 4.8 \S$ & $5.9 \pm 6.6 \S$ & $3.7 \pm 6.5 \S$ & $2.2 \pm 3.7 \S$ & $<0.001$ \\
\hline Vitality (\%) & $76.1 \pm 9.0$ & $18.2 \pm 11.0$ & $27.2 \pm 12.1$ & $19.7 \pm 11.4$ & $15.4 \pm 10.1$ & $<0.001$ \\
\hline Morp (\%) & $17.8 \pm 3.9$ & $10.2 \pm 3.4$ & $12.7 \pm 3.8$ & $10.6 \pm 3.7$ & $9.2 \pm 3.3$ & $<0.001$ \\
\hline
\end{tabular}

Conc=concentration, Total mot=total motility, PR=progressive motility, Morp=sperm morphology, S.D.=standard deviation, mM=millimolar, $\mathrm{mL}=$ milliliter, $\S$ Data were not normally distributed, *Significance of differences between fresh semen samples, and post-thawed semen samples of each group 
Cryopreservation resulted in a significant reduction in sperm motility parameters. Progressive motility had a mean value of $50.6 \%$, before freezing and a mean value of $5.9 \%, 3.7 \%, 2.2 \%$ and $3.2 \%$ after cryopreserved, with $50 \mathrm{mM}$ trehalose, $100 \mathrm{mM}$ trehalose, $200 \mathrm{mM}$ trehalose and $50 \mathrm{mM}$ sucrose, respectively $(p-v a l u e<0.001)$. Reflecting the total number of motile spermatozoa, progressive motility plus non-progressive motility, was also considered. This value decreased significantly after the frozen-thawed process ( $p$-value $<0.001$ ). Comparing with different cryoprotectants, the best post-thaw sperm parameters were obtained within the $50 \mathrm{mM}$ trehalose group.

Figure 1 shows the percentages of difference, between mean of both total and progressive sperm motility, from 124 cryopreserved semen samples comparing between $50 \mathrm{mM}$ sucrose, and three different trehalose concentrations. The sperm motility was significantly higher after $50 \mathrm{mM}$ trehalose, than it was after $50 \mathrm{mM}$ sucrose for both total motility and progressive motility ( $p$-value $<$
0.001 and $p$-value $=0.037$ ), respectively. To compare 50 $\mathrm{mM}$ sucrose, there were no significant differences regarding the statistically compared post-thaw sperm motility frozen in $100 \mathrm{mM}$, and $200 \mathrm{mM}$ trehalose.

The percentages of difference between; mean of post thawed sperm vitality frozen in $50 \mathrm{mM}$ sucrose and three different trehalose concentrations are presented in Figure 2. Sperm vitality, which was determined using the eosin-nigrosin staining method, revealed a mean value of $76.1 \%$ before freezing. This value diminished to $27.2 \%$, $19.7 \%, 15.4 \%$ and $18.2 \%$, after cryopreserved with $50 \mathrm{mM}$ trehalose, $100 \mathrm{mM}$ trehalose and $200 \mathrm{mM}$ trehalose and $50 \mathrm{mM}$ sucrose, respectively. After cryopreserved in $50 \mathrm{mM}$ trehalose, the percentages of differences between mean of post-thawed sperm vitality were significantly better than $50 \mathrm{mM}$ sucrose ( $p$-value<0.001), however, post-thawed sperm vitality were not different in both $100 \mathrm{mM}$ trehalose, and $200 \mathrm{mM}$ trehalose, when compared with $50 \mathrm{mM}$ sucrose.

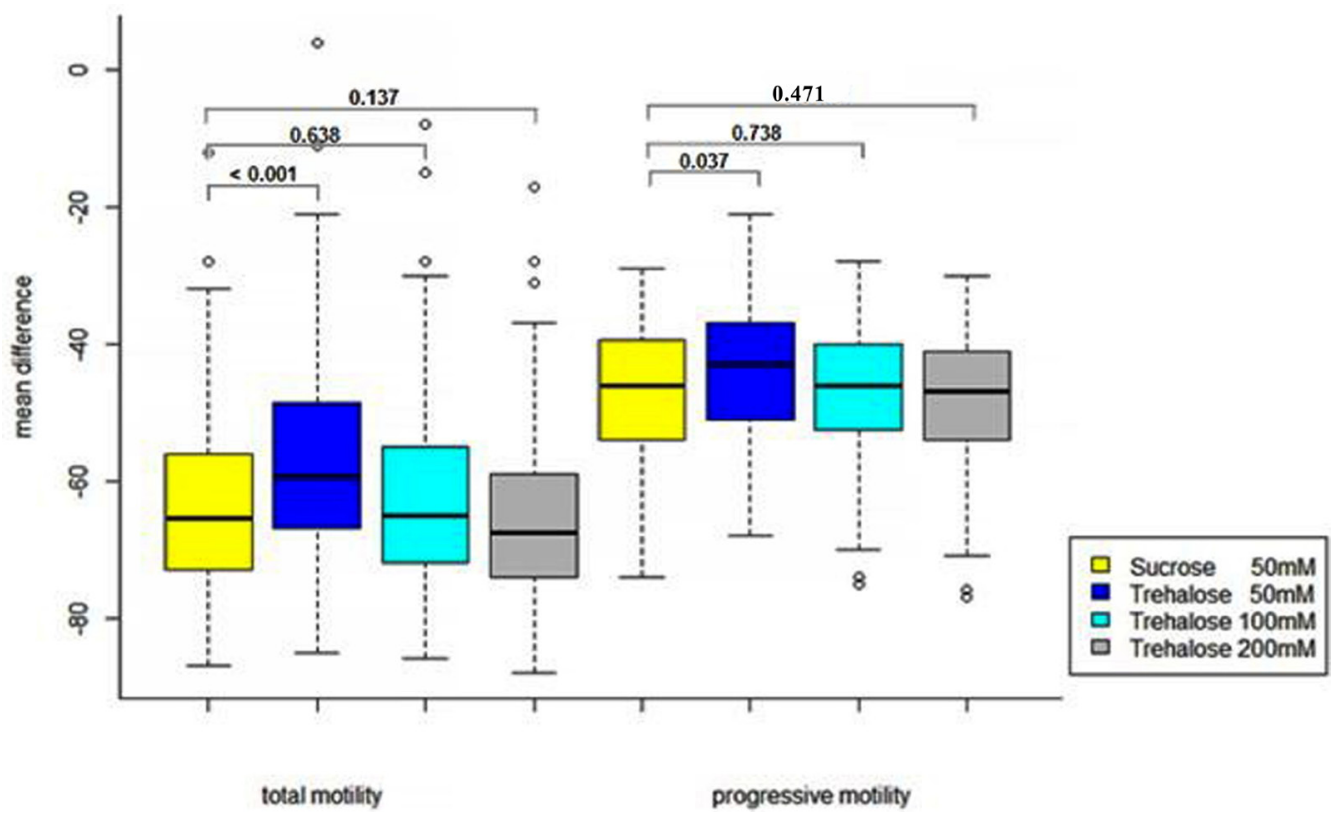

Figure 1 The percentage of difference between; mean of post thawed total sperm motility and progressive motility frozen in 50 milimolar sucrose, and three different trehalose concentrations. 


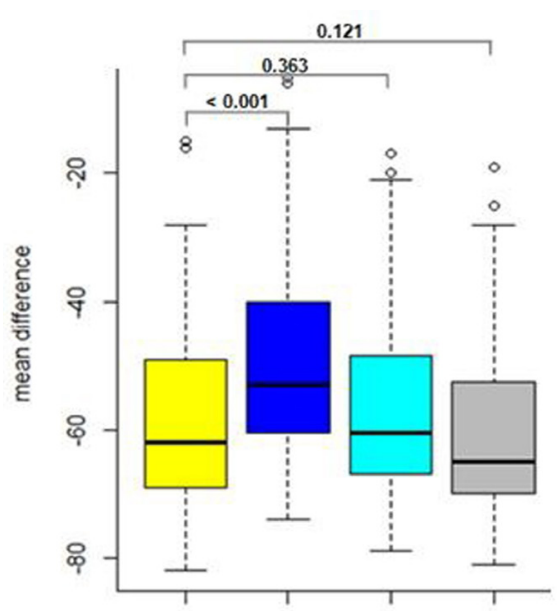

sperm vitality

Figure 2 The percentage of difference between; mean of post thawed sperm vitality frozen in 50 milimolar sucrose, and three different trehalose concentrations.

Assessment of sperm morphology, using light microscopy, revealed a statistically significant decrease in the percentage of normal spermatozoa, after thawing, in that there was a mean value of $17.8 \%$ before freezing, and a mean value of $12.7 \%, 10.6 \%, 9.1 \%$ and $10.2 \%$ after cryopreserved, with $50 \mathrm{mM}$ trehalose, $100 \mathrm{mM}$ trehalose, $200 \mathrm{mM}$ trehalose and $50 \mathrm{mM}$ sucrose ( $\mathrm{p}$-value $<0.001)$. Figure 3 shows the percentages of differences between, mean of post-thawed morphology. The sperm cryopreserved in $50 \mathrm{mM}$ trehalose were significantly better than that of $50 \mathrm{mM}$ sucrose ( $p$-value $<0.001$ ) in post-thawed morphology, but no significant differences in both $100 \mathrm{mM}$, and $200 \mathrm{mM}$ trehalose comparing with $50 \mathrm{mM}$ sucrose were found.

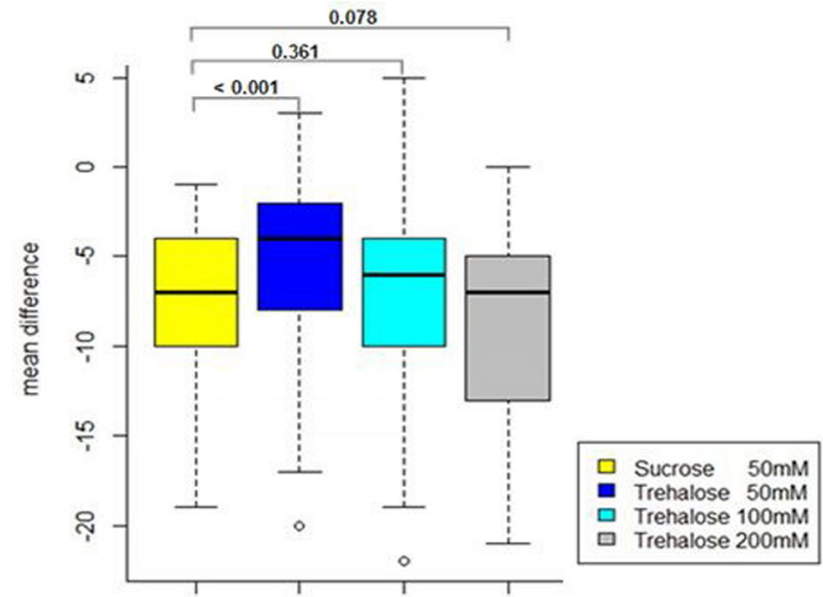

sperm morphology

Figure 3 The percentage of difference between: mean of post thawed sperm morphology frozen in 50 milimolar sucrose, and three different trehalose concentrations.

\section{Discussion}

Our study showed that only $50 \mathrm{mM}$ of trehalose has better post-thawed sperm parameters than sucrose, and other trehalose concentrations. Although animal studies showed the benefits of trehalose over sucrose in sperm freezing, only a few studies have investigated the effects of trehalose on human sperm cryopreservation.,

Cryopreservation adversely affected the cryosurvival of human spermatozoa. Unsurprisingly, in our study, progressive motility, total motility, vitality and morphology of spermatozoa; after the frozen-thawed process, was significantly reduced when compared with the fresh semen values. These deleterious effects on spermatozoa were similar to results obtained in a previous study. ${ }^{13}$ 
Only supplementation of $50 \mathrm{mM}$ trehalose led to a significant increase in the post-thawed human sperm parameters, including sperm motility, vitality and morphology, compared with other higher trehalose concentrations in our study. This demonstrated that the osmolarity in the cryopreservation medium is of importance to sperm parameters. Higher concentrations of trehalose, over $50 \mathrm{mM}$, may cause the osmolarity to become too high, and can produce substantial osmotic damage, and again this is consistent with another, previous study ${ }^{3,12}$; whereas the osmolarity has a significant effect on human sperm motility. Gao et al. ${ }^{14}$ suggested that appropriate osmolarity of the cryopreservation medium is between 250 and 350 milliosmoles per liter. However, our study did not determine osmolarity of the medium.

To compare between trehalose and sucrose, the study also showed the benefits of using trehalose, over sucrose, at the same concentrations. These beneficial effects have also been reported in animal studies conducted on buffalo, boar, goat, and mouse. ${ }^{4-6,15}$ Although sucrose, and trehalose are also a non-reducing disaccharide, they are significantly different in their structures. The bonding of trehalose has a clam shell conformation, which has more stability than those of the more open conformation of sucrose. ${ }^{15,16}$ The key as to why trehalose showed more of a benefit, over sucrose, in the cryopreservation process may be the $\alpha, \alpha-(1->1)$ glycosidic linkage of trehalose. ${ }^{16}$ In vivo studies demonstrated that: the $\alpha, \alpha$-trehalose binds with the cell's lipid membrane and forms solid, supported lipid bilayers. ${ }^{15-18}$ This helps to maintain cell integrity as well as decreasing cell damage during the cryopreservation process. ${ }^{8,16,18}$ In contrast, Liu et al. ${ }^{3}$ showed that trehalose and sucrose were both effective, while our study found that trehalose was significantly better than sucrose in terms of recovering post-thaw's sperm motility. This discrepancy result may be that our study added glycerol in the freezing medium, which reported a synergic effect of trehalose on cell integrity. ${ }^{8}$

One strength of our study is that the large number of human semen samples used, which were then compared between trehalose and sucrose as a non-permeating cryoprotectant. However, the study was limited by the fact that sperm parameters (motility, vitality and morphology), that were used in this study, may not accurately predict the invitro fertilization outcome (IVF). ${ }^{19}$ A systematic review $^{20}$ in the evaluation of sperm function has been recommended using the hemizona assay, for the prediction of IVF outcomes.

\section{Conclusion}

In conclusion, the usage of trehalose, instead of sucrose, as a non-permeating cryoprotectant improved post-thawed human sperm parameters, with a concentration of $50 \mathrm{mM}$ trehalose demonstrating the best sperm parameters. However, further investigations may be needed to determine the optimal concentration of trehalose, or types of cryoprotectant to achieve the best quality of sperm after cryopreservation. In addition, studies to evaluate the effective of trehalose, for patients presenting with oligospermia, would be more useful.

\section{Acknowledgement}

This study was supported by the Faculty of Medicine, Prince of Songkla University. The authors thank all volunteers, for donating their semen samples in this study. We thank Professor Tippawan Liabsuetrakul, of the Epidemiology Unit, for her assistance in statistical analysis; Suwadee Wirotkarun for her assistance in the preparations of sperm freezing media; and Associated Professor Jitti Hanprasertpong for his insightful comments on the paper. 


\section{Conflict of interest}

The authors have no conflict of interest to declare.

\section{References}

1. Sadeghi MR. It is time to pay more attention to sperm cryopreservation: now more than ever!. J Reprod Infertil 2016;17:1

2. Stanic P, Tandara M, Sonicki Z, Simunic V, Radakovic B, Suchanek E. Comparison of protective media and freezing techniques for cryopreservation of human semen. Eur J Obstet Gynecol Reprod Biol 2000;91:65-70.

3. Liu J, Tanrikut C, Wright DL, Lee GY, Toner M, Biggers JD, et al. Cryopreservation of human spermatozoa with minimal non-permeable cryoprotectant. Cryobiology 2016;73:162-7.

4. Shiva Shankar Reddy N, Jagan Mohonarao G, Atreja SK. Effects of adding taurine and trehalose to a tris-based egg yolk extender on buffalo (Bubalus bubalis) sperm quality following cryopreservation. Anim Reprod Sci 2010;119:18390.

5. Malo C, Gil L, Gonzalez N, Cano R, de Blas I, Espinosa E. Comparing sugar type supplementation for cryopreservation of boar semen in egg yolk based extender. Cryobiology 2010; $61: 17-21$.

6. Thompson KA, Richa J, Liebhaber SA, Storey BT. Dialysis addition of trehalose/glycerol cryoprotectant allows recovery of cryopreserved mouse spermatozoa with satisfactory fertilizing ability as assessed by yield of live young. J Androl 2001;22:339-44.

7. Aboagla EM, Terada T. Trehalose-enhanced fluidity of the goat sperm membrane and its protection during freezing. Biol Reprod 2003;69:1245-50.

8. Aisen EG, Medina VH, Venturino A. Cryopreservation and post-thawed fertility of ram semen frozen in different trehalose concentrations. Theriogenology 2002;57:1801-8.

9. World Health Organization. WHO laboratory manual for the examination and processing of human semen. $5^{\text {th }}$ ed. Geneva: WHO; 2010.

10. Kruger TF, Acosta AA, Simmons KF, Swanson RJ, Matta JF,
Oehninger S. Predictive value of abnormal sperm morphology in in vitro fertilization. Fertil Steril 1988;49:112-7.

11. Hammadeh ME, Greiner S, Rosenbaum P, Schmidt W. Comparison between human sperm preservation medium and TEST-yolk buffer on protecting chromatin and morphology integrity of human spermatozoa in fertile and subfertile men after freeze-thawing procedure. J Androl 2001;22:1012-8.

12. Schulz M, Risopatron J, Matus G, Pineda E, Rojas C, Isachenko $\mathrm{V}$, et al. Trehalose sustains a higher post-thaw sperm motility than sucrose in vitrified human sperm. Andrologia 2017;49:1-3.

13. Ozkavukcu S, Erdemli E, Isik A, Oztuna D, Karahuseyinoglu S. Effects of cryopreservation on sperm parameters and ultrastructural morphology of human spermatozoa. J Assist Reprod Genet 2008;25:403-11.

14. Gao DY, Liu J, Liu C, McGann LE, Watson PF, Kleinhans FW, et al. Prevention of osmotic injury to human spermatozoa during addition and removal of glycerol. Hum Reprod 1995; 10:1109-22.

15. Storey BT, Noiles EE, Thompson KA. Comparison of glycerol, other polyols, trehalose, and raffinose to provide a defined cryoprotectant medium for mouse sperm cryopreservation. Cryobiology 1998;37:46-58.

16. Albertorio F, Chapa VA, Chen X, Diaz AJ, Cremer PS. The $\alpha, \alpha-(1->1)$ linkage of trehalose is key to anhydrobiotic preservation. J Am Chem Soc 2007;29:10567-74.

17. Anchordoguy TJ, Rudolph AS, Carpenter JF, Crowe JH. Modes of interaction of cryoprotectants with membrane phospholipids during freezing. Cryobiology 1987;24:324-31.

18. Crowe JH. Anhydrobiosis: an unsolved problem with applications in human welfare. Subcell Biochem 2015;71:263-80.

19. Bastiaan HS, Menkveld R, Oehninger S, Franken DR. Zona pellucida induced acrosome reaction, sperm morphology, and sperm-zona binding assessments among subfertile men. J Assist Reprod Genet 2002;19:329-34.

20. Vogiatzi P, Chrelias C, Cahill DJ, Creatsa M, Vrachnis N, Iliodromiti Z, et al. Hemizona assay and sperm penetration assay in the prediction of IVF outcome: a systematic review. Biomed Res Int 2013;2013. doi: 10.1155/2013/945825. 